\title{
A Single Case of Two Synchronous Metastatic Tumours
}

\author{
Filipa Pereira, Tiago Alpoim, Maria Leitão, Manuela Machado* \\ Medical Oncology Department, IPO Porto, Porto, Portugal \\ Email: *m.machado.fn@gmail.com
}

How to cite this paper: Pereira, F., Alpoim, T., Leitão, M. and Machado, M. (2019) A Single Case of Two Synchronous Metastatic Tumours. Journal of Cancer Therapy, 10, 671-676. https://doi.org/10.4236/jct.2019.108055

Received: July 9, 2019

Accepted: August 18, 2019

Published: August 21, 2019

Copyright $\odot 2019$ by author(s) and Scientific Research Publishing Inc. This work is licensed under the Creative Commons Attribution International License (CC BY 4.0).

http://creativecommons.org/licenses/by/4.0/

(c) (i) Open Access

\begin{abstract}
The incidence of multiple primary tumours increases with age and due to multiple other factors as environmental and genetic predisposition. The authors describe a case of a 77-year-old female patient with diagnosis in September 2009 of colon mucinous adenocarcinoma. She was submitted to surgery, considered R1 and adjuvant chemotherapy. Six months after treatment she had abdominal recurrence on a nodular lesion in peritoneal fat. The patient was submitted to surgery and did multiple courses of systemic treatment. She underwent cytoreductive surgery with hyperthermic chemotherapy, too. In December 2014 she presented a nodular lesion on the bladder and after excision, the histology confirmed a high-grade transitional cell carcinoma and she maintains treatment with endovesical BCG instillations. After that, she had no evidence of peritoneal disease progression. Besides the bad prognosis associated to peritoneal disease, our case report describes a long survival (5 years with no disease progression) in a patient with high risk at diagnosis to peritoneal recurrence. This case shows the importance of multimodal approach and the impact of locoregional treatment in localized metastatic disease.
\end{abstract}

\section{Keywords}

Colon Cancer, Bladder Cancer, Multiple Primary Neoplasms Synchronous

\section{Introduction}

The incidence of multiple primary tumours increases with age and there are currently more cases reported, not only because of advances in treatment that lead to an increased in overall survival, but also because of better accuracy of diagnostic modalities that increases the rate of diagnosis. In addition, the rise of life expectancy, immunosuppression, viral infections, environmental factors and 
genetic predisposition are potential additional factors for multiple carcinogenesis. Still, the risk factors like tobacco cannot be forgotten.

The authors describe a case of a patient with recurrent peritoneal metastasis of mucinous adenocarcinoma of ascending colon, submitted to multimodal treatment with stable disease after 5 years and a transitional cell bladder carcinoma that is currently with no evidence of disease.

\section{Case}

A 77-year-old female patient, with a medical history of hypertension, obesity and active smoking, was submitted in September 2009 to a right hemicolectomy due to mucinous adenocarcinoma of ascending colon. The tumour was staged as pT3 N0 M0, with the surgery considered R1, due to a positive radial surgical margin.

In multidisciplinary team, she was proposed to perform complementary chemotherapy (ChT). The patient initiated mFOLFOX6 (5-fluorouracil, folinic acid and oxaliplatin) scheme in November 2009. Due to oxaliplatin neurotoxicity, the dose of that drug was reduced by $25 \%$ after the third cycle. In February 2010, because of a grade 3 hypersensitivity reaction, oxaliplatin was discontinued and she maintained treatment with DeGramont (5-fluorouracil plus folinic acid) scheme to a total of 12 cycles (the last one in May 2010).

In December 2010, as a result of persistent abdominal pain, an abdominopelvic computed tomography scan (CT) and a magnetic resonance imaging (MRI) were done; both showed a single nodular lesion with $2.5 \mathrm{~cm}$ in peritoneal fat, near the ileocolic anastomosis, very suspicious of metastatic disease.

In a multidisciplinary team, the patient was proposed to a segmental enterectomy, which was performed in March 2011. The histological examination revealed the presence of a mucinous adenocarcinoma implant in the adipose tissue, with no lymph node involvement and a proximal circumferential resection margin smaller than $1 \mathrm{~cm}$. The tumour presented a RAS mutation. Then, she was proposed to palliative ChT and started treatment on May 2011 with FOLFIRI (5-Fluorouracil, Folinic Acid and Irinotecan) scheme. After 12 cycles (October 2011), she presented stable disease. Due to gastrointestinal toxicity, irinotecan was reduced by $25 \%$ after the 1 st cycle. After the 12th cycle, ChT was stopped by the patient' will. Peritoneal disease progression was documented on March 2012 on CT scan and the patient' resume FOLFIRI plus bevacizumab. She did treatment (12 cycles) until September 2012 with partial response as the better response. Again, ChT was stopped by the patient' will.

After 3 months on surveillance, in December 2012, a new disease progression was documented as peritoneal carcinomatosis. Positron-emission tomographycomputed tomography (PET-CT) revealed 18F-FDG hypermetabolism in two indistinguishable colon masses in the hepatic angle, one of which in contact with the abdominal wall as well as another with abdominal wall invasion. Because of progressive disease only in peritoneum, the clinical case was discussed in mul- 
tidisciplinary team and the patient was proposed to a cytoreductive surgery with hyperthermic chemotherapy (HIPEC) with mitomycin C, which was done in February 2013. The anatomopathological examination revealed implants involving the transverse colon, sigmoid colon and left para-rectal area as well as the left ovary; Twenty-eight lymph nodes were isolated, 4 of which metastasized. The patient continued treatment with DeGramont scheme, and 5 months later, in July 2013, as a result of abdominal pain, a new thoraco-abdominopelvic CT was carried out and it was suspicious of new peritoneal recurrence, given the appearance of a $10 \mathrm{~mm}$ nodular densification anterior to the inferior vena cava, and an area of irregular densification in the anterior abdominal wall. To better clarify these findings, it was performed a PET-CT, which revealed hypercaptation in the suspected CT lesions. She started FOLFIRI again, and after 12 cycles she had partial response on CT scan.

In February 2014, a PET-CT response evaluation was performed and revealed hypercaptation in a single abdominal tumour implant. The patient underwent exploratory laparotomy in March 2014, with resection of two abdominal nodules, one located in the mesentery anterior to the inferior vena cava and another in the sigmoid mesocolon. Histology of the mesentery implant confirmed a mucinous adenocarcinoma metastasis, whereas the other nodule revealed cytosteatonecrosis. Given the poor tolerance to $\mathrm{ChT}$, it was decided to maintain clinical surveillance.

Nine months later, in December 2014, on a CT scan, the patient presented a nodular image with $12.5 \mathrm{~mm}$ on the left posterior wall of bladder and another nodular lesion suspicious in the right lobe of the liver with $11 \mathrm{~mm}$. On the PET-CT, a 18FDG hypercaptation focus located on the posterior wall of the bladder in probable relation with malignant tumour was observed, with no other abnormal uptake suggesting metabolic activity.

The patient underwent transurethral resection of bladder tumour (TUR-BT) in March 2015, being resected two neoformations of $2-3 \mathrm{~cm}$ each. Mitomycin C instillation was performed in the immediate postoperative period. The histological examination revealed a high-grade transitional cell carcinoma without chorion invasion ( $\mathrm{pTa}$ ).

In May 2017, the patient did a cystoscopy that showed a local recurrence ofbladder tumour. Patient underwent a new transurethral resection and histological exam confirmed a low-grade papillary urothelial carcinoma, with invasion of the chorion ( $\mathrm{p}$ T1 N0 M0). Then, it was continued treatment with endovesical BCG instillations, which are currently being performed, with no evidence of relapse.

The patient has been presented since 2014 with no evidence of new peritoneal disease progression, with stability of findings in multiple revaluation imagiologic techniques, that have been showing persistence of right mesenteric nodular lesion with $2 \mathrm{~cm}$ of maximum diameter, unspecific and stable, described during the last 5 years; the last thoraco abdominopelvic CT was performed in May 2019 
and and a MRI in June 2019.

\section{Discussion}

After a curative treatment of the primary colon cancer, $20 \%-30 \%$ will develop locoregional or systemic recurrence during the follow-up. The liver is the most common metastatic site, followed by peritoneal cavity, which has a big impact on prognosis. About half of patients with CRC will have disease recurrence as peritoneal metastasis and $25 \%$ of these will have peritoneal disease as the only site of metastatic disease. Median OS in metastatic CRC is 30 months, with improvement of therapeutic options, and up to $20 \%$ of patients treated with ChT are alive at 5-years. The colon cancer with peritoneal carcinomatosis prognosis is extremely poor [1] [2].

The present case represents an example of metachronous peritoneal metastatis from primary colon cancer, with a long survival after multimodal approach, namely with HIPEC.

The introduction of aggressive cytoredutive procedures combined with intraperitoneal ChT promised to alter the prognosis of these patients. Some studies showed a benefit in OS with 5-year survival rates $20 \%-30 \%$ [3]. In a study, patients with metachronous peritoneal disease showed a median OS of 28 months with this approach [4]. Aggressive surgical cytoreduction with intraperitoneal chemotherapy has been applied to patients with isolated peritoneal carcinomatosis, but the benefits of this approach remain controversial. The date remains insufficient to conclude about if the survival advantage is due to treatment or tumor biologic features. Another question about HIPEC is the importance of skills and experience of surgeon, so this procedure needs to be done in a high-experience center [5] [6] [7]. Results of PRODIGE 7 show a similar overall post-operative mortality and morbidity rates between 2 groups (with or without HIPEC). Median recurrence free survival was 11.1 months in non-HIPEC group and 13.21 months in HIPEC group ( $p$ 0.486). The median OS was not different too. At median follow up of 64 months, OS was 41.2 months in non-HIPEC group and 41.7 months in HIPEC group (p 0.995), showing that the addition of HIPEC did not given a benefit in metastatic CRC outcomes [8].

Peritoneal disease is diagnosed in advanced stage in most of patients. Laparotomy or laparoscopy is the gold standard to diagnosis metachronous peritoneal metastasis but is difficult to perform given this is an invasive option. CT has a low sensitivity for little lesions $(<5 \mathrm{~mm})$. MRI and PET-CT show a better sensitivity and a better correlation with intraoperatively disease [9] [10]. However, given the difficult to diagnosis there are trials that try to support second-look surgery in high risk patients. Segelman et al. considered as risk factors to peritoneal disease: tumors in right colon, emergency surgery, surgery non-R0, incomplete lymphadenectomy ( $<12$ nodes examined) [11]. Other risk factors described are: T4 disease, perforated cancer, ovarian metastases, and positive peritoneal fluid cytology [10]. Some authors concluded that prophylac- 
tic HIPEC or second-look surgery after systemic treatment in high risk patients would anticipate clinical or radiological relapse, treating carcinomatoses in early stages, when cure is possible [10] [11] [12].

Besides the bad prognosis associated do peritoneal disease, our case report describes a long survival (5 years with no disease evidence) in a patient with high risk at diagnosis to peritoneal recurrence (right colon, R1 surgery, mucinous carcinoma). This case shows the importance of multimodal approach and the impact of locoregional treatment in localized metastatic disease. Another important question in this case is the importance of biopsy of recurrent disease. In an era of target therapy, the biopsy of metastasis had another importance to decide relatively to treatment. In addition, this case illustrates that another primary tumor could be differential diagnosis and we must think about this possibility, essentially in case of presence of risk factors.

\section{Conflicts of Interest}

The authors declare no conflicts of interest regarding the publication of this paper.

\section{References}

[1] Manfredi, S., Bouvier, A.M., Lepage, C., Hatem, C., Dancourt, V. and Faivre, J. (2006) Incidence and Patterns of Recurrence after Resection for Cure of Colonic Cancer in a Well Defined Population. British Journal of Surgery, 93, 1115-1122. https://doi.org/10.1002/bjs.5349

[2] Mise, Y., Zimmitti, G., Shindoh, J., et al. (2015) RAS Mutations Predict Radiologic and Pathologic Response in Patients Treated with Chemotherapy before Resection of Colorectal Liver Metastases. Annals of Surgical Oncology, 22, 834-843. https://doi.org/10.1245/s10434-014-4042-6

[3] Witkamp, A.J., de Bree, E., Kaag, M.M., Boot, H., Beijnen, J.H., van Slooten, G., et al. (2001) Extensive Cytoredutive Surgery Followed by Intra-Operative Hyperthermic Intraperitoneal Chemotherapy with Mitomycin-C in Patients with Peritoneal Carcinomatosis of Colorectal Origin. European Journal of Cancer, 37, 979-984. https://doi.org/10.1016/S0959-8049(01)00058-2

[4] Jayne, D.G., Fook, S., Loi, C. and Seow-Choen, F. (2002) Peritoneal Carcinomatosis from Colorectal Cancer. British Journal of Surgery, 89, 1545-1550. https://doi.org/10.1046/j.1365-2168.2002.02274.x

[5] Elias, D., Gilly, F., Boutitie, F., et al. (2010) Peritoneal Colorectal Carcinomatosis Treated with Surgery and Perioperative Intraperitoneal Chemotherapy: Retrospective Analysis of 523 Patients from a Multicentric French Study. Journal of Clinical Oncology, 28, 63-68. https://doi.org/10.1200/JCO.2009.23.9285

[6] Yan, T.D., Zappa, L., Edwards, G., et al. (2007) Perioperative Outcomes of Cytoreductive Surgery and Perioperative Intraperitoneal Chemotherapy for Non-Appendiceal Peritoneal Carcinomatosis from a Prospective Database. Journal of Surgical Oncology, 96, 102-112. https://doi.org/10.1002/jso.20736

[7] Simkens, G.A., van Oudheusden, T.R., Braam, H.J., et al. (2016) Treatment-Related Mortality after Cytoreductive Surgery and HIPEC in Patients with Colorectal Peritoneal Carcinomatosis Is Underestimated by Conventional Parameters. Annals of Surgical Oncology, 23, 99-105. https://doi.org/10.1245/s10434-015-4699-5 
[8] Quenet, F., Elias, D., Roca, L., Goéré, D., Ghouti, L., Pocard, M., et al. (2019) A UNICANCER Phase III Trial of Hyperthermic Intra-Peritoneal Chemotherapy (HIPEC) for Colorectal Peritoneal Carcinomatosis. PRODIGE 7. European Journal of Surgical Oncology, 45, e17. https://doi.org/10.1016/j.ejso.2018.10.086

[9] Chang, M.C., Chen, J.H., Liang, J.A., Huang, W.S., Cheng, K.Y. and Kao, C.H. (2013) PET or PET/CT for Detection of Peritoneal Carcinomatosis: A Meta-Analysis. Clinical Nuclear Medicine, 38, 623-629. https://doi.org/10.1097/RLU.0b013e318299609f

[10] Jacobson, R., Sherman, S.K., Dadaleh, F. and Turaga, K.K. (2018) Peritoneal Metastases in Colorectal Cancer. Annals of Surgical Oncology, 25, 2145-2151. https://doi.org/10.1245/s10434-018-6490-x

[11] Segelman, J., Akre, O., Gustafsson, U.O., Bottai, M. and Martling, A. (2016) External Validation of Models Predicting the Individual Risk of Metachronous Peritoneal Carcinomatosis from Colon and Rectal Cancer. Colorectal Disease, 18, 378-385. https://doi.org/10.1111/codi.13219

[12] Elias, D., Honoré, C., Dumont, F., Ducreux, M., Boige, V. and Malka, D. (2011) Results of Systematic Second-Look Surgery Plus HIPEC in Asymptomatic Patients Presenting a High Risk of Developing Colorectal Peritoneal Carcinomatosis. Annals of Surgery, 254, 289-293. https://doi.org/10.1097/SLA.0b013e31822638f6 